\title{
Asociaciones entre rendimiento en comprensión de textos y estrategias pedagógicas utilizadas por docentes
}

\section{Asociaciones entre comprensión lectora y estrategias docentes}

\author{
Lorena Canet Juric \\ María Laura Andrés \\ Isabel Introzzi \\ María Richard's
}

\begin{abstract}
Resumen
El objetivo de este trabajo ha sido explorar las asociaciones entre el rendimiento en comprensión lectora y distintas estrategias docentes utilizadas con alumnos de segundo y tercer grado de Educación Primaria Básica (EPB) de la Ciudad de Mar del Plata (Argentina). El supuesto de este estudio afirma que existe una asociación entre el rendimiento en comprensión lectora y las estrategias pedagógicas docentes. Se aplicó una adaptación del PROLEC para evaluar el rendimiento en lectura y un cuestionario construido para explorar las estrategias docentes. Los instrumentos fueron administrados a una muestra aleatoria de alumnos de $2^{\circ}$ y $3^{\circ}$ año de 10 escuelas municipales $(n=258)$ y a sus respectivos docentes $(n=64)$. Los resultados permitieron aportar evidencia empírica sobre la ausencia de asociaciones estadísticamente significativas entre ambas variables, lo que permite suponer que la lectoescritura es un fenómeno complejo que depende de numerosos factores como las diferencias cognitivas individuales, el contexto alfabetizador, etc. Palabras clave: comprensión lectora; estrategias pedagógicas; educación primaria pública.
\end{abstract}

\section{Associações entre rendimento na compreensão de textos e estratégias pedagógicas utilizadas por docentes}

\section{Resumo}

O objetivo deste trabalho foi explorar as associações entre o rendimento em compreensão de leitura e diferentes estratégias docentes utilizadas em alunos da segunda e terceira série do ensino fundamental da cidade de Mar del Plata (Argentina). O pressuposto deste estudo afirma que existe uma associação entre o rendimento em compreensão de leitura e as estratégias pedagógicas docentes. Foi aplicado uma adaptação do PROLEC para avaliar o rendimento em leitura e um questionário elaborado para explorar as estratégias docentes. Os instrumentos foram aplicados em uma amostra aleatória de alunos da $2^{\mathrm{a}}$ e $3^{\mathrm{a}}$ série de 10 escolas municipais $(n=258)$ e nos seus respectivos docentes $(n=64)$. Os resultados permitiram contribuir com evidência empírica a respeito da ausência de associações estadisticamente significativas entre ambas as variáveis, o que permite supor que a leitura-escrita é um fenômeno complexo que depende de vários fatores, como ser as diferenças cognitivas individuais, o contexto de alfabetização, etc.

Palavras-chave: compreensão em leitura; estratégias pedagógicas; ensino fundamental público.

\section{Associations between achievement in reading understanding and pedagogical strategies used by teachers}

\begin{abstract}
The aim of this work has been to explore the relationships between reading comprehension achievement and strategies used by teachers in second and third class of public schools of Mar del Plata City. The assumption of this study affirms that exist an association between reading comprehension achievement and strategies used by teachers. It was applied an adaptation of PROLEC to evaluate reading comprehension achievement and a questionnaire to explore the educational strategies. The instruments were administered to a random sample of students of $2^{\circ}$ and $3^{\circ}$ year of 10 public schools $(n=258)$ and their respective teachers $(n=64)$. The outcomes allows contribute empirical evidence of the absence of statistically significant associations between both variables which allows to suppose that reading comprehension is a complex phenomenon that depends on numerous factors like individual cognitives differences and familiar support.
\end{abstract}




\section{Introducción}

Leer no se reduce a decodificar las palabras sino que también significa comprender el mensaje escrito de un texto. Sin embargo, para conseguirlo resulta necesario, aunque no suficiente, el automatismo de los procesos de decodificación que genera la liberación de recursos atencionales (Perfetti, 1985, 199I). De acuerdo a la literatura científica, se supone que la comprensión lectora es un complejo proceso que genera una representación integrada y coherente del texto por parte del lector. La mayor parte de las investigaciones sobre dificultades en la lectura considera que las fallas en el proceso de decodificación es el problema principal que enfrenta un sujeto para la comprensión lectora. Sin embargo, el déficit de comprensión no puede ser atribuido exclusivamente a fallas en la decodificación, sino que hay un conjunto de factores que, sin duda, también tienen influencia: la confusión respecto a las demandas de la tarea, la pobreza de vocabulario, los escasos conocimientos previos, los problemas de memoria, la falta de entrenamiento en estrategias de comprensión, el escaso control de la comprensión o las diferencias en el modo de enseñar a buenos y malos lectores (Citoler, 2000).

Según Defior Citoler (2000), existen una serie de investigaciones (Allington, 1983; Biemiller, 1977-1978) que demuestran que los malos lectores reciben menos enseñanza en comprensión y más en decodificación que los buenos lectores. Aunque suene extraño, estos estudios han permitido corroborar de forma empírica que los malos lectores no solamente recibían menos cantidad de instrucción en comprensión, sino que se les exigía demasiado tiempo práctica de la lectura oral con continuas interrupciones para corregir errores pero sin proponer reflexiones en relación al contenido del texto. La frecuente interrupción genera en los niños la sensación de que deben depositar en otra persona la tarea de evaluar su ejecución lectora, generando un déficit de las habilidades de control de la exactitud y de la comprensión lectora.

Comprender el lenguaje requiere de dos niveles de procesamiento de los contenidos de una oración: el sintáctico y el semántico. Una oración es un conjunto de palabras que guardan entre sí determinadas relaci- ones sintácticas y para comprenderlas hay que identificarlas y vincularlas entre sí. Comprender una oración es conferirle un significado, procesar semánticamente su contenido como un todo. De acuerdo a Molinari Marotto (2000) "un texto no es un conjunto aleatorio de oraciones, sino un conjunto coherente" (p.l19) y, por lo tanto, la comprensión del texto implica establecer relaciones que mantengan unidas las ideas, para que sea percibido y representado en la memoria como una estructura coherente.

Además de distinguir entre comprensión de textos y de oraciones, es necesario establecer diferencias entre los distintos tipos textuales. Según la clasificación de Brewer (1980) se los denomina textos expositivos, narrativos y descriptivos. Cada uno de ellos requiere la aplicación de distintas estrategias y modalidades pedagógicas de enseñanza.

Los textos narrativos incluyen personajes y acciones, acontecimientos desencadenantes y ocurrencia de sucesos en cadena temporal, relaciones causales y motivacionales, finales como consecuencia de una acción; el objetivo de los mismos es entretener y además poseen objetivos literarios estéticos (Molinari Marotto, 2000; Braslavsky, 2005). Para fomentar la comprensión en cuentos narrativos el docente puede realizar las siguientes actividades: Antes de la lectura: proponer una discusión sobre sus propósitos; generar hipótesis derivadas del título, realizar ilustraciones, conocer el nombre del autor, etc.; Durante la lectura: generar y responder preguntas, buscar el significado de las palabras que no resultan familiares; y Después de la lectura: revivir el cuento a través de la renarración oral, hacer dibujos y dramatizaciones, etc. (Braslavsky, 2005).

Los textos expositivos describen conceptos de tipo abstracto y relaciones del tipo lógico con el fin de explicar, informar, persuadir. Estos textos deben ser, según Slater y Graves (1989): informativos, explicativos, orientativos e incluir la narración. Este tipo de texto informa sobre investigaciones, datos, personas, hechos, teorías, etc. Para que el alumno los comprenda, el docente debe incitarlo a tener un plan estratégico de comprensión y debe ayudarlo a reconocer cómo se diferencia este de otros tipos de texto (Braslavsky, 2005). 
La estrategia general más frecuentemente aplicada por el docente con el objetivo de motivar la comprensión lectora es la realización de preguntas explícitas o inferenciales en relación al texto (Sánchez, Orrantia \& Rosales, 1992). Un texto contiene más información de la que aparece explícitamente enunciada y es tarea del lector completar con sus conocimientos las lagunas que presenta (Davoudi, 2004; Borzone, Rosemberg, Diuk, Silvestre \& Plana, 2004). La realización de inferencias ayuda a los lectores a construir y dar significados al texto, sin ellas sería prácticamente imposible comprender el texto a un nivel más macro que el de la oración (Paris, Wasik, \& Turner, 1991; García Madruga et al., 1999; Cain \& Oakhill, 2004). Numerosas investigaciones han demostrado que los niños tienen más dificultades en responder preguntas inferenciales que literales o explícitas (Paris et al, |99I).

Según Geva (1985), los lectores hábiles aplican sistemáticamente varias estrategias de lectura como la paráfrasis, la solución de problemas y el repaso. Existe un acuerdo generalizado sobre la posibilidad de modelar diversas estrategias o habilidades y la importancia de su cesión gradual del control al alumno, para que recupere su autonomía (Schmitt \& Baumann, 1989; Grimes, 2004; Sánchez, Orrantia \& Rosales, 1992; del Río, 1985). De acuerdo a esto, se infiere la importancia de enseñar de forma sistemática estrategias que favorezcan la comprensión lectora.

Teniendo en cuenta lo expuesto, este trabajo se propuso evaluar la relación entre las estrategias pedagógicas y la comprensión lectora en alumnos de $2^{\circ}$ y $3^{\circ}$ año de la EPB, bajo el supuesto de que es factible encontrar niveles más altos de comprensión en aquellos alumnos que han recibido una formación y un entrenamiento sistemático en estrategias de lectura..

\section{Método}

\section{Participantes}

Se trabajó con 64 docentes (64 mujeres, promedio de años de ejercicio en la docencia $=17)$, de $2^{\circ}$ año $(n=33)$ y de $3^{\circ}$ año $(n=31)$, y con 298 de sus estudiantes (162 mujeres y 136 varones) pertenecientes a segundo grado $(n=146)$ y tercer grado $(n=152)$. La edad promedio fue de 7.57 años en segundo grado y de 8.60 años en tercer grado. La totalidad de la muestra fue seleccionada al azar mediante un registro áulico de 10 Escuelas Municipales de la ciudad de Mar del Plata, Argentina.

\section{Instrumentos}

Para evaluar la comprensión lectora se utilizó la versión adaptada del PROLEC (Andrés, Canet Juric, Introzzi y Urquijo, 2006). Las pruebas fueron administradas en forma individual durante los últimos dos meses del año lectivo. A los fines de este estudio, se consideraron las siguientes escalas:

Escala Lectura de Palabras y Pseudopalabras. Esta prueba contiene 60 estímulos (20 palabras de alta frecuencia, 20 palabras de baja frecuencia y 20 pseudopalabras, en los tres casos la mitad son de longitud corta y la otra mitad de longitud larga). Permite verificar si los alumnos tienen automatizados los procesos léxicos.

Escala de Comprensión de Textos. Incluye dos textos breves, uno narrativo y otro expositivo, con cuatro preguntas cada uno, dos expositivas y dos narrativas. Permite evaluar la comprensión lectora a nivel textos (ver ANEXO A).

\section{Cuestionario para el docente}

Para explorar las estrategias docentes, se confeccionó un cuestionario que fue aplicado en forma individual a cada docente. Del cuestionario mencionado se consideró la pregunta referida a las actividades de comprensión aplicadas por el docente.

I- Qué actividades utiliza para el refuerzo de los aprendizajes de la lectoescritura.

2- Describa que actividades utiliza para que los alumnos comprendan los textos que leen.

3- iHa recibido alguna capacitación específica sobre enseñanza de comprensión lectora?

4- ¿Cómo considera usted que debiera reforzarse la comprensión de textos?

Adicionalmente, para completar esta información se analizaron las actividades utilizadas por el docente a 
través de la observación sistematizada de los cuadernos de los alumnos mediante una grilla de observación (ver ANEXO B), donde el examinador registraba las tareas de comprensión lectora observadas (Urquijo, Canet Juric, Andrés y Ané, 2006).

\section{Procedimiento}

Las entrevistas fueron realizadas individualmente con los docentes en el aula o algún lugar de la escuela destinado para tal fin. Los niños también fueron evaluados de manera individual en un lugar tranquilo de la escuela, se solicitó el consentimiento por escrito de los padres para tal fin.

\section{Análisis estadístico}

Para facilitar y resumir los resultados se construyeron tres índices en base a la clasificación de estrategias utilizada por Braslavsky (2005): Índice de antes de la lectura (reactivar conocimientos previos, vocabulario, predicciones o anticipaciones sobre el texto, ); Índice de durante la lectura (lectura, ordenar secuencias, estructura del texto, modelado de lectura, completar oraciones con las palabras que faltan, comprensión de instrucciones, ) e Índice de después de la lectura (preguntas sobre lo leído, renarración del texto en forma oral o escrita, dibujar sobre lo leído, debate oral sobre el texto, cambiar el final, responder verdadero o falso, unir con flechas, respuestas de opción múltiple). Para la comprensión de textos se tomó la escala de comprensión de textos del PROLEC (Andrés, Canet Juric, Introzzi \& Urquijo, 2006). Final- mente, se analizaron las relaciones entre cada uno de los índices elaborados y los niveles de comprensión de textos, utilizando la Prueba de Independencia de Jicuadrado $\left(\chi^{2}, \mathrm{pd} \leq 05\right)$.

\section{Resultados}

\section{Resultados PROLEC}

En la Tabla I se observa que el nivel de rendimiento en lectura presenta variaciones entre el $2^{\circ}$ y el $3^{\circ}$ curso, produciéndose un incremento en este último. El porcentaje de acierto en estas pruebas es alto en general., sin embargo los niveles de comprensión lectora son bajos tanto en segundo como en tercer año, presentando un porcentaje de acierto sólo del $54.9 \%$ en segundo y $59.62 \%$ en tercero. En general, podemos afirmar que el desempeño en las tres pruebas de lectura es similar, siendo menor el desempeño en lectura de pseudopalabras y en lectura de palabras infrecuentes que en lectura de palabras frecuentes.

En ambos cursos se presenta un mejor rendimiento en lectura de palabras y una disminución en el rendimiento en comprensión en general, sobre todo en comprensión de textos. En general, se observa una mejora de todas las habilidades en tercero.

Se observa un desempeño superior en la variable comprensión de oración en los alumnos de $3^{\circ}$ año $(M=$ $5.21)$ que en la variable comprensión de textos $(M=$ 4.76), presentándose mayores rendimientos en ambas pruebas en alumnos de tercer grado.

Tabla I. Estadísticos descriptivos de cantidad de aciertos en lectura y comprensión discriminados por curso para toda la muestra

\begin{tabular}{llccccc}
\hline Año & Variable & $\mathrm{N}$ & Mínimo & Máximo & Media & $\begin{array}{c}\text { Desv. } \\
\text { típica }\end{array}$ \\
\hline \multirow{2}{*}{2} & $\begin{array}{l}\text { Lectura de Palabras frecuentes } \\
\begin{array}{l}\text { Lectura de Palabras } \\
\text { infrecuentes }\end{array}\end{array}$ & 146 & 0,00 & 20,00 & 18,28 & 4,02 \\
& Lectura de Pseudopalabras & 146 & 0,00 & 20,00 & 17,69 & 3,92 \\
& $\begin{array}{l}\text { Lectura de Palabras frecuentes } \\
3\end{array}$ & 152 & 0,00 & 20,00 & 19,24 & 2,12 \\
$\begin{array}{l}\text { Lectura de Palabras } \\
\text { infrecuentes }\end{array}$ & 152 & 2,00 & 20,00 & 18,55 & 2,40 \\
& Lectura de Pseudopalabras & 152 & 1,00 & 20,00 & 17,80 & 2,61 \\
\hline
\end{tabular}


Para poder realizar un análisis de las asociaciones entre el rendimiento en comprensión de textos y las estrategias usadas por los docentes, se seleccionaron aquellas escuelas que mostraron los rendimientos más altos y aquellas otras que mostraron los más bajos en Comprensión de textos. Para esto se tuvieron en cuenta los puntajes medios de la totalidad de la muestra de escuelas municipales en Comprensión de textos $\left(M 2^{\circ}\right.$ año $=4,33, M 3^{\circ}$ año $\left.=4,7 I\right)$. Se seleccionaron aquellas escuelas que se encontraban un desvío estándar por encima y por debajo de la media de la muestra normativa (ver Tabla 2). Posteriormente, se incluye un análisis de cuáles son las estrategias pedagógicas que utilizan los docentes de estas escuelas.

\section{Resultados cuestionarios}

Estrategias utilizadas por los docentes para que los alumnos comprendan los textos que leen en $2^{\mathrm{do}}$ y $3^{\text {er }}$ grado.

En segundo grado se observa que existe toda una franja de estrategias que son utilizadas por las escuelas con puntaje alto y no son usadas por las escuelas con puntajes bajas (Comprensión de consignas y puesta en común, Expresión oral y escrita, Completar oraciones, Relectura, Relato de cuentos y Debate). A su vez, las escuelas consideradas dentro de las de bajos rendimiento, utilizan estrategias que no son utilizadas por las otras escuelas (Reconocer tipos textuales, Unir con flechas, Vocabulario, Predicciones sobre el texto, Modelado, Escritura de oraciones, Respuesta de opción múltiple y Completar oraciones)

En tercer grado se puede observar de manera similar a lo que ocurre en segundo grado (ver Tabla 4), ya que existe toda una franja de estrategias que son utilizadas por las escuelas con puntaje alto $y$ que no son usadas por las escuelas con puntajes bajos (Dictado, Reglas ortográficas, Separar en sílabas, Ordenar alfabéticamente, Familias de palabras, Producciones escritas de los alumnos, Dramatizaciones, Libros a casa). A su vez, las escuelas consideradas dentro de las de bajos rendimiento, utilizan estrategias que no son utilizadas por las otras escuelas (Debate, Modelado,

Tabla 2. Resultados de comprensión lectora de $2^{\text {do }}$ grado discriminados por medias y desvíos altos y bajos de 4 escuelas municipales

\begin{tabular}{cccc}
\hline \multirow{2}{*}{ Pontuación } & \multirow{2}{*}{ Escuelas } & \multicolumn{2}{c}{ Comprensión de textos } \\
\cline { 3 - 4 } & & $\mathrm{M}$ & $\mathrm{SD}$ \\
\hline \multirow{2}{*}{ Altas } & 5 & 5.24 & 1.71 \\
& 8 & 6.13 & 0.88 \\
\multirow{3}{*}{ Bajas } & 4 & 3.44 & 2.27 \\
& 6 & 2.25 & 2.62 \\
\hline
\end{tabular}

Tabla 3. Resultados de comprensión lectora de $3^{\text {ro }}$ grado discriminados por medias y desvíos altos y bajos de 4 escuelas municipales

\begin{tabular}{cccc}
\hline \multirow{2}{*}{ Pontuación } & \multirow{2}{*}{ Escuelas } & \multicolumn{2}{c}{ Comprensión de textos } \\
\cline { 3 - 4 } & & $\mathrm{M}$ & $\mathrm{SD}$ \\
\hline \multirow{2}{*}{ Altas } & 16 & 5.73 & 1.55 \\
& 8 & 6.13 & 1.06 \\
\multirow{3}{*}{ Bajas } & 11 & 4.00 & 2.92 \\
& 6 & 4.16 & 1.81 \\
\hline
\end{tabular}


Tabla 4. Comparación de frecuencias de estrategias enunciadas por los docentes entre escuelas con altos y bajos puntajesen segundo grado

\begin{tabular}{lcccc}
\hline \multirow{2}{*}{ Estrategias } & \multicolumn{2}{c}{ Escuelas con puntajes altos } & \multicolumn{2}{c}{ Escuelas con puntajes bajos } \\
\cline { 2 - 5 } Lectura & 8 & 5 & 4 & 6 \\
Preguntas & 2 & 0 & 2 & 2 \\
V o F & 2 & 1 & 3 & 1 \\
Renarración & 1 & 0 & 1 & 0 \\
Comprensión de consignas & 1 & 0 & 0 & 1 \\
Expresión oral y escrita & 1 & 0 & 0 & 0 \\
Completar oraciones & 1 & 0 & 0 & 0 \\
Relato de cuentos & 1 & 0 & 1 & 2 \\
Ordenar secuencias & 1 & 0 & 1 & 0 \\
Dibujar & 1 & 1 & 1 & 1 \\
Comentarios orales sobre lo leído & 0 & 1 & 1 & 0 \\
Debate & 1 & 1 & 1 & 3 \\
Reconocer tipos textuales & 1 & 1 & 0 & 0 \\
Unir con flechas & 0 & 0 & 1 & 0 \\
Vocabulario & 0 & 0 & 1 & 0 \\
Predicciones sobre el texto & 0 & 0 & 1 & 2 \\
Modelado & 1 & 0 & 0 & 1 \\
Escritura de oraciones & 0 & 0 & 1 & 1 \\
Respuesta de opción múltiple & 0 & 0 & 0 & 1 \\
\hline
\end{tabular}

Cambiar el final de cuentos, Búsqueda de sinónimos y antónimos, Relacionar con conocimientos previos).

Asociación entre el rendimiento en comprensión lectora y estrategias docentes.

Las medias más altas en comprensión de textos no presentan asociaciones estadísticamente significativas con un tipo particular de estrategias docentes. Índice $3 \chi^{2}(3,19)=3.026, \mathrm{p}>=.05$; Índice $2 \chi^{2}(5$, $19)=4.93, \mathrm{p}>=.05$ e Índice $3 \chi^{2}(7,19)=8.74$, $\mathrm{p}>=.05$.

Un dato relevante es que las estrategias docentes incluídas en el İndice 3 de Después de la lectura presentan una media más alta $(M=2,16)$ en comparación con el resto de las medias pertenecientes a los restantes índices (Índice I $M=0,40$; Índice $2 M=1,20$ ).

\section{Discusión}

El elevado rendimiento en las escalas de lectura de palabras y pseudopalabras indica que los participantes han automatizado los procesos léxicos. Por lo tanto, las puntuaciones bajas en las tareas que evalúan la comprensión, indicarían que estas dificultades no se deben a un déficit en los procesos léxicos, sino que se encuentran relacionados a otras variables. Existen un conjunto de factores que tienen influencia en el rendimiento de esta variable, a saber: la confusión respecto a las demandas de la tarea, la pobreza de vocabulario, los escasos conocimientos previos, los problemas de memoria, la falta de entrenamiento en estrategias de comprensión, el escaso control de la comprensión o las diferencias en el modo de enseñar a buenos y malos lectores 
Tabla 5. Comparación de frecuencias de estrategias enunciadas por los docentes entre escuelas con altos y bajos puntajesen tercero grado

\begin{tabular}{lcccc}
\hline \multirow{2}{*}{ Estrategias } & Escuelas con puntajes altos & Escuelas con puntajes bajos \\
\cline { 2 - 5 } & 16 & 8 & 11 & 6 \\
\hline Lectura & 1 & 2 & 1 & 0 \\
Preguntas & 1 & 1 & 2 & 1 \\
Renarración & 0 & 0 & 0 & 1 \\
Ordenar secuencias & 0 & 0 & 1 & 1 \\
Dibujar & 1 & 0 & 1 & 0 \\
Comentarios orales sobre lo leído & 1 & 1 & 2 & 1 \\
Vocabulario & 1 & 0 & 0 & 1 \\
Predicciones sobre el texto & 1 & 0 & 1 & 1 \\
Modelado & 0 & 1 & 1 & 0 \\
Completar oraciones & 0 & 0 & 1 & 0 \\
Cambiar el final de cuentos & 0 & 0 & 1 & 0 \\
Búsqueda de sinónimos y antónimos & 0 & 0 & 0 & 1 \\
Relacionar con conocimientos previos & 0 & 0 & 0 & 1 \\
Vo F & 1 & 0 & 0 & 0 \\
\hline
\end{tabular}

(Citoler, 2000, Allington, 1983; Biemiller, 19771978). Mostrar la existencia de estas diferencias constituía el objetivo de este trabajo.

Al analizar la comprensión de oraciones se observó que los alumnos presentan rendimiento superior en la comprensión de oraciones que de textos. Esta actividad, si bien resulta compleja, presenta menos dificultad de procesamiento ya que el alumno sólo se debe preocupar del procesamiento individual de las oraciones, sin necesidad de realizar operaciones más complejas como las de integración en unidades superiores, actividad que sí es requerida para la comprensión global del texto.

Nuestros resultados no han permitido obtener evidencia empírica confirmatoria a favor de la asociación entre el rendimiento en comprensión de textos y las estrategias pedagógicas usadas por los docentes.

En coincidencia con algunos autores (Paris, Wasik, \& Turner, 1991; García Madruga, Elosúa, Gutiérrez, Luque \& Garate, 1999 y Cain \& Oakhill, 2004 ) los resultados demostraron que una de las estrategias mayormente elegidas por los docentes para favorecer la comprensión del texto es elaborar preguntas sobre el texto para que los alumnos posteriormente las respondan. Otra estrategia enunciada como importante para la comprensión, fue la práctica de la lectura, quizás esto tenga que ver con el manejo de una teoría intuitiva por parte del docente en relación a la importancia de automatizar los procesos de decodificación y acceso léxico para así poder liberar recursos atencionales y ponerlos al servicio de procesos cognitivos más profundos, en este caso, al servicio de la comprensión.

En general, no hay diferencias cuantitativas importantes en relación a las estrategias enunciadas por los docentes, sólo se observa mención de distintas estrategias en las escuelas que presentan buenos rendimientos y en las escuelas que presentan bajos rendimientos. Estas estrategias, tanto en un tipo de rendimiento como en otro, son enunciadas por el docente en forma aislada y no como un paquete de estrategias a ser utilizado en distintos momentos de la lectura. Es decir, que lo que se ha podido observar 
es más bien la falta de sistematización y organización en el planeamiento de la enseñanza de la comprensión lectora. Se utilizan un número elevado de diversas estrategias pero éstas no presentan un ordenamiento lógico entre sí, o algún tipo de criterio clasificatorio que permita agruparlas y prever un entrenamiento en la comprensión textual, más bien se trata de una especie de abanico en que se incluyen multiplicidad de estrategias.

Más allá de los resultados relacionados con los cuestionarios docentes, el hecho de que el docente enuncie una actividad no constituye un indicador de la frecuencia de uso de las mismas, lo que podría haber sido de mayor utilidad para la posterior comparación entre docentes.

Consideramos que es necesario incluir otro tipo de mediciones o registros en estudios futuros que incluyan información más detallada acerca de las estrategias docentes tales como: la frecuencia de uso de las mismas, cantidad de horas dedicas a la instrucción en comprensión lectora, horas asignadas a tareas escolares que se relacionen con la comprensión lectora, etc.

\section{Bibliográfia}

Andrés, M. L., Canet Juric, L. \& Introzzi, I. (2006). Modificación de una prueba para evaluar los procesos intervinientes en la Lectoescritura. Mesa Redonda. Em I Encuentro Nacional de Evaluación Psicológica y Educativa (pp. 36-39). Avances Teóricos y Técnicos de la Medición Psicológica, Córdoba, Argentina.

Allington, R. L. (1983). The reading instruction provided to readers of differing reading abilities. Elementary Scholl Journal, 83, 548-559.

Biemiller, A. (1977-1978). Relationships between oral reading rates for letters, words, and simple text in the development of reading achievement. Reading Research Quarterly, 13, 223-253.

Borzone, A. M., Rosemberg, C. R., Diuk, B., Silvestri, A., \& Plana, D. (2004). Niños y maestros por el camino de la alfabetización. Buenos Aires: Red de apoyo escolar.

Bravslavsky, B. (2005). Enseñar a entender lo que se lee. La alfabetización en la familia y en la escuela. Buenos Aires: Fondo de Cultura Económica.
Brewer, W. F. (1980). Theoretical sigues in reading comprehension. Hillsdale, New Jersey: Lawrence Erlbaum Associates. Em C. Molinari Marotto (Ed.), Introducción a los Modelos Cognitivos de la Comprensión del Lenguaje. Buenos Aires: Eudeba.

Cain, K., \& Oakhill, J. (2004). Children's reading comprehension ability: Concurrent prediction by working memory, verbal ability, and component skills. Journal of Educational Psychology, $96,3|-4|$.

Davoudi, M. (2004). Inference generation skill and text comprehension. The Reading Matrix, (5), I06-I23.

Defior Citoler, S. (2000). Las dificultades del aprendizaje: un enfoque cognitivo. Lectura, escritura y matemáticas. ( $2^{\mathrm{a}}$ ed.). Granada: Educación para la diversidad.

Del Río, P. (1985). Investigación y Práctica educativa en el desarrollo de la comprensión lectora. Infancia y Aprendizaje, 3I-32, 32-44.

García Madruga, J. A., Eloúsa, M. R., Gutiérrez, F., Luque, J. L., \& Gárate, M. (1999). Comprensión Lectora y Memoria Operativa. Aspectos evolutivos e instruccionales. Barcelona: Paidós.

Geva, E. ( 1 985). Mejora de la comprensión lectora mediante diagramas de flujo. Infancia y Aprendizaje, 3I-32, 45-66.

Grimes, S. (2004). The search of meaning. How you can boost kids' reading comprehension. Scholl Library Journal, 50(5), 48-52.

Molinari Marotto, C. (2000). Introducción a los Modelos Cognitivos de la Comprensión del Lenguaje. Buenos Aires: Eudeba.

Paris, S. G., Wasik, B. A., \& Turner J. C. (199I). The development of strategic readers. Em P. D. Pearson, R. Barr, M. L. Kamil \& P. Mosenthal (Eds.), Handbook of reading research (pp. 609-640, v. I). New York: Longman.

Perfetti, C. A. (1985). Reading Ability. New York: Oxford University Press.

Perfetti, C. A. (I99I). Representations and awareness in the acquisition of reading competence. Em L. Rieben \& C. A. Perfetti (Eds.), Learning to read: Basic research and its implications (pp. 33-44). Hillsdale, NJ: Lawrence Erlbaum Associates.

Sánchez, E., Orrantia, J., \& Rosales, J. ( 1992). Cómo mejorar la comprensión de textos en el aula. Comunicación, Lenguaje y Educación, 14, 89-112.

Schmitt, C. M., \& Baumann, J. (1989). Cómo incorporar las estrategias de control de la comprensión a la enseñanza con textos base de lectura. Comunicación, Lenguaje y Educación, I, 45-50. 
Slater, W. H., \& Graves, M. F. (1989). Research on expository text: implications for teachers. Em K. D. Bluth (Ed.), Children's Comprehension of text. Newark: International Reading Association.
Urquijo, S., Canet Juric, L., Andrés, M. L., \& Ané, A. (2006). Los Procesos Lectores en Primer Ciclo de EGB (Informe Técnico para la Municipalidad de General Pueyrredón). Mar del Plata, Buenos Aires: Universidad Nacional de Mar del Plata, Grupo de Psicología Cognitiva y Educacional.

Recebido em: 06/08/2007

Revisado em: 05//2/2007

Aprovado em 29/0I/2008

Sobre as autoras

Lorena Canet Juric, (Icanetjuric@gmail.com) - doutoranda em Psicologia pela Universidade Nacional de Sao Luis. Trabaja en el Centro de Investigación en Procesos Básicos, Metodología y Educación, en la Universidade Nacional do Mar del Plata y es Becaria Doctoral de Investigación do Consejo Nacional de Investigaciones Científicas y técnicas y docente de la Facultad de Psicologia. (CONICET).

María Laura Andrés (malauandres@yahoo.com.ar) - Universidad Nacional de Mar del Plata. Trabaja en el Centro de Investigación en Procesos Básicos, Metodología y Educación, en la Universidade Nacional do Mar del Plata. Es Becaria de Iniciación en esta Universidade.

Isabel Introzzi (isaintrozzi@hotmail.com) - doutoranda em Psicología pela Universidade Nacional de Sao Luis. Trabaja en el Centro de Investigación en Procesos Básicos, Metodología y Educación, en la Universidade Nacional do Mar del Plata y es Becaria Doctoral de Investigación do Consejo Nacional de Investigaciones Científicas y técnicas y docente de la Facultad de Psicologia. (CONICET).

María Richard's (mariamartarichards@gmail.com) - doutoranda em Psicología pela Universidade Nacional de Sao Luis. Trabaja en el Centro de Investigación en Procesos Básicos, Metodología y Educación, en la Universidade Nacional do Mar del Plata y es Becaria Doctoral de Investigación do Consejo Nacional de Investigaciones Científicas y técnicas y docente de la Facultad de Psicologia. (CONICET).

Nota das autoras

Este trabajo es resultado de la Investigación efectuada por el Centro de Procesos Básicos, Metodología y Educación (en ese entonces Grupo de Psicología Cognitiva y Educacional) en el marco del proyecto: Alfabetización y Psicología Cognitiva dirigido por el doctor Sebastián Urquijo. 


\section{ANEXO A}

Texto Narrativo

ERA EL CUMPLEAÑOS DE MARISA Y ALLÍ ESTABAN TODAS SUS AMIGAS ESPERANDO A QUE EMPEZARA LA FIESTA. DE REPENTE, OYERON UN RUIDO EN LA COCINA Y CUANDO ENTRARON VIERON QUE EL GATO HABÍA TIRADO LA TORTA. MARISA SE PUSO MUY TRISTE PORQUE YA NO PODÍA APAGAR LAS VELAS. PERO EN ESE MOMENTO LLEGÓ SU PADRINO CON UNA GRAN TORTA DE REGALO Y TODOS SE PUSIERON MUY CONTENTOS.

Preguntas

I- ¿QUÉ ESTABAN ESPERANDO LAS AMIGAS DE MARISA?

2- ¿QUÉ ERA EL RUIDO QUE OYERON EN LA COCINA?

3- ¿POR QUÉ NO PODRÍA MARISA APAGAR LAS VELAS?

4- ¿QUÉ TRAJO EL PADRINO DE MARISA?

Texto Expositivo

A LA ORILLA DE LOS RÍOS CRECE UN ÁRBOL MUY BONITO DE FLORES AMARILLAS LLAMADO SAUCE. LOS SAUCES TIENEN UNAS RAMAS MUY LARGAS Y CON MUCHAS HOJAS POR LO QUE DAN UNA BUENA SOMBRA. SU TRONCO ES LARGO Y FLEXIBLE Y SE UTILIZA PARA FABRICAR BASTONES. EN LA ÉPOCA DE VERANO QUE LA TIERRA ESTÁ SECA, SUS RAÍCES SE ALARGAN MUCHOS METROS BUSCANDO EL AGUA.

Preguntas

I- ¿DÓNDE CRECEN LOS SAUCES?

2- ¿POR QUÉ DAN BUENA SOMBRA LOS SAUCES?

3- ¿PARA QUÉ SE UTILIZA SU TRONCO?

4- ¿CÓMO SON SUS RAÍCES? 


\section{ANEXO B}

\section{Trilla de observación}

\begin{tabular}{|l|l|}
\hline \multicolumn{1}{|l|}{ De uso exclusivo para el entrevistador) } \\
\multicolumn{1}{|l|}{$1-$ Ambientación/decoración del aula: marque con una cruz lo que observa } \\
\hline 71 & Nombres de los alumnos \\
\hline 72 & Palabras con sus respectivos dibujos debajo \\
\hline 73 & Letras sueltas decoradas \\
\hline \multicolumn{2}{|l|}{} \\
\hline \multicolumn{2}{|l|}{ 2- } \\
\hline 81 & Actividades observadas en los cuadernos: \\
\hline 82 & Correcritura de palabras ortográfica mediante repetición \\
\hline 83 & Armado de palabras con sílabas sueltas \\
\hline 84 & Armado de oraciones con sílabas sueltas \\
\hline 85 & Búsqueda de palabras en sopas de letras y sílabas \\
\hline 86 & Segmentación de palabras en una oración \\
\hline 87 & Ordenamiento alfabético de palabras \\
\hline 88 & Escritura de oraciones en distintos formatos de letras \\
\hline 89 & Segmentación de palabras en sílabas \\
\hline 810 & Recorte de imágenes de revistas y escritura del nombre \\
\hline 811 & Dictado \\
\hline 812 & Lectura de textos breves/cuentos/rimas \\
\hline 813 & Rearmado de cuentos \\
\hline 814 & Cumplimiento de consignas de dibujo y reconocimiento \\
\hline 815 & Realización de hipótesis sobre el título \\
\hline 816 & Armado de secuencias con oraciones desordenadas \\
\hline 817 & Identificación de oraciones representativas \\
\hline 818 & Búsqueda del significado de palabras por contexto \\
\hline 819 & Búsqueda de palabras en el diccionario \\
\hline 820 & Rellenado de frases extraídas de un cuento \\
\hline 821 & Realización de preguntas explí́citas e implícitas sobre un cuento \\
\hline 822 & Reflexión sobre la comprensión del texto (escuela 5) \\
\hline 823 & Identificación de tipos textuales \\
\hline 824 & Búsqueda de palabras que contengan determinadas letras solas o combinadas \\
\hline & \\
\hline & \\
\hline
\end{tabular}

